\title{
PLASTIC BEHAVIOR OF PREDEFORMED ICE CRYSTALS*
}

\author{
By D. M. Joncich,
}

(Department of Physics and Materials Research Laboratory, University of Illinois at UrbanaChampaign, Urbana, Illinois 6r8or, U.S.A.)

\section{J. Holder}

(Department of Geology and Materials Research Laboratory, University of Illinois at Urbana-Champaign, Urbana, Illinois 6r $80 \mathrm{r}$, U.S.A.)

\section{and A. V. Granato}

(Department of Physics and Materials Research Laboratory, University of Illinois at UrbanaChampaign, Urbana, Illinois 6r 80 I, U.S.A.)

Abstract. Conventional studies of plastic deformation have been complicated by the simultaneous variation of dislocation velocity and dislocation density during the tests. In the present study this difficulty was avoided by carrying out deformation tests at low stress levels on samples which had been predeformed to relatively higher stresses prior to the measurements. Creep, mechanical after effect, constant strain-rate, and stress relaxation tests were carried out as a function of measurement stress or strain-rate, temperature, and predeformation level. The results were analysed in terms of a linear stress-dependent dislocation velocity in order to determine whether that simple behavior is able to account for the macroscopic deformation behavior of ice crystals. This report is a brief summary of the results; a complete discussion is to be published elsewhere. The principal results of the study are as follows:

(I) The observed behavior was particularly simple for predeformed samples. The creep strain showed a nearly linear increase with time, without the large positive curvature characteristic of conventional tests. No stress maxima were observed in the constant strain-rate tests as have been found in previous studies of non-predeformed samples. The (complete) stress relaxation curve was virtually identical in shape to the inverted constant strain-rate curve.

(2) The steady-state creep and constant strain-rate behavior could be described to good approximation in terms of the motion of a constant density of dislocations moving with the same linear stress-dependent velocities as have been observed directly by others. The strainrates were linear in stress and the estimated dislocation densities $\left(4\right.$ to $\left.16 \times 10^{5} \mathrm{~cm}^{-2}\right)$ varied with the magnitude of the predeformation level in a manner consistent with previous observations. The strain-rates or stress levels are exponential in $\mathrm{I} / \mathcal{T}$ with an activation energy of $0.6 \mathrm{eV}$, which is approximately equal to the activation energy reported for the motion of dislocations and for the mechanical relaxation time in internal friction studies.

(3) A small transient creep behavior, and a small but measurable mechanical after-effect with the same $\left(\approx_{3} \mathrm{~min}\right)$ time constant were present. These effects, as well as the nonexponential behavior of the constant strain-rate and stress-relaxation stress-strain results, could be accounted for by including a second, anelastic, component in a deformation model corresponding to a restoring force in addition to the linear viscous drag force on dislocations. This leads to a differential equation which is linear in stress and strain, but involves both firstand second-order time derivatives.

(4) The solutions of this differential equation describe the observed mechanical response well, and provide a general internal consistency check for the model.

* This research was supported in part by the National Science Foundation under Grants DMR-76-0 1058 and DMR-77-10556. 
(5) A quantitative fit of the experimental test results to the dislocation model gives values of $3-4$ for the ratios of total dislocation density to the recoverable component and values of 7-8 dyn $/ \mathrm{cm}^{2}$ for the restoring force constant for the recoverable dislocations. The restoring force constants and recoverable dislocation densities were, within experimental error, found to be independent of temperature, measurement stress and strain-rate, and predeformation level. Values found for the parameters for creep and mechanical after-effect tests were equal within experimental error to the values found from constant strain-rate and stress-relaxation tests carried out on the same sample. If the recoverable dislocation component is identified as bowed-out dislocation segments whose ends are fixed, the restoring force could be accounted for by the elastic line tension of dislocation segments of lengths of about $8 \times \mathrm{IO}^{-3} \mathrm{~cm}$.

No feature of the experimental results was inconsistent with this dislocation model, and the results of the study are all in agreement with the theory proposed by Weertman in which the dislocation drag force is very large because of the stress-induced ordering of water molecules in the stress field of the moving dislocation.

This paper is to be submitted for publication in full in another journal.

\title{
DISGUSSION
}

N. K. Sinha: During the tests I found it difficult to maintain the imposed strain-rate to a constant level. It was also observed that it was impossible to maintain the imposed strain to a constant level in a stress-relaxation test. These are because of the inherent elasticity of ordinary testing machines. Were the experiments on stress relaxation and strain-rate tests performed with a closed-loop testing machine? If not, the experimental results may represent only the response of the ice-machine system.

J. Holder: The tests were carried out in an MTS testing machine, in a closed loop configuration. All strains were monitored by capacitor plates attached direct to the specimen (as opposed to the cross-head).

J. Perez: You have used the Orowan relation; did you take into consideration, at least at higher temperatures, dynamic recovery phenomena leading to values of $\Lambda$ depending on stress and on temperature in the steady state?

Holder: No systematic study of dislocation density as a function of predeformation stress was carried out; our concern was to maintain a constant density during the measurements after the predeformation. Our control in this regard was to compare strain-rates during the actual tests to the strain-rates measured during the predeformation. The densities determined by the equation were the same, within our uncertainty.

\section{EFFECT OF HYDROSTATIC PRESSURE ON THE CREEP OF ICE}

\author{
By Stephen J. Jones
}

(Glaciology Division, Inland Waters Directorate, Department of Fisheries and Environment, Ottawa, Ontario $\mathrm{K}_{\mathrm{I}} \mathrm{A}$ oE 7 , Canada)

Abstract. Uniaxial compressive creep tests on single crystals and polycrystals of ice at about $-\mathrm{IO}^{\circ} \mathrm{C}$ under different hydrostatic pressures are described. After creeping under a constant load at atmospheric pressure for some hours, a hydrostatic pressure of the order of $35 \mathrm{MN} \mathrm{m}^{-2}$ was applied and the change in strain-rate was noted. Some hours later the 